\title{
LRRK2 p.M1646T is associated with glucocerebrosidase activity and with Parkinson's disease
}

Yuri L. Sosero, MD, ${ }^{1,2}$ Eric Yu, BSc, ${ }^{1,2}$ Lynne Krohn, MSc, ${ }^{1,2}$ Uladzislau Rudakou, MSc, ${ }^{1,2}$ Kheireddin Mufti, MSc, ${ }^{1,2}$ Jennifer A. Ruskey, MSc, ${ }^{1,3}$ Farnaz Asayesh, MSc, ${ }^{1,3}$ Sandra B. Laurent, BSc, ${ }^{3}$ Dan Spiegelman, MSc, ${ }^{1}$ Stanley Fahn, MD, ${ }^{4}$ Cheryl Waters, MD, ${ }^{4}$ S. Pablo Sardi, PhD, ${ }^{5}$ International Parkinson Disease Genomics Consortium (IPDGC), Sara Bandres-Ciga, PhD, ${ }^{6}$ Roy N. Alcalay, MD, MSc, ${ }^{4,7}$ Ziv Gan-Or, MD, PhD, ${ }^{1,2,3}$ Konstantin Senkevich, MD, PhD ${ }^{1,3 *}$

1. Montreal Neurological Institute, McGill University, Montréal, QC, H3A 1A1, Canada

2. Department of Human Genetics, McGill University, Montréal, QC, H3A 1A1, Canada

3. Department of Neurology and neurosurgery, McGill University, Montréal, QC, H3A 0G4, Canada, Canada

4. Department of Neurology, College of Physicians and Surgeons, Columbia University Medical Center, New York, NY, USA.

5. Rare and Neurological Diseases Therapeutic Area, Sanofi, Framingham, MA, USA.

6. Molecular Genetics Section, Laboratory of Neurogenetics, National Institute on Aging, National Institutes of Health, Bethesda, MD 20892, USA

7. Taub Institute for Research on Alzheimer's Disease and the Aging Brain, College of Physicians and Surgeons, Columbia University Medical Center, New York, NY, USA.

\section{*Corresponding author:}

Konstantin Senkevich Montreal Neurological Institute, McGill University 1033 Pine Avenue, West, Ludmer Pavilion, room 308-309 Montreal, QC, H3A 1A1, Phone: +1-438-979-5052

e-mail: konstantin.senkevich@mcgill.ca

Text word count: 1728 words

Running title: $L R R K 2$ variants modify GCase activity

Keywords: Parkinson's disease; GBA; Glucocerebrosidase; LRRK2 
medRxiv preprint doi: https://doi.org/10.1101/2020.09.23.20197558; this version posted September 24, 2020. The copyright holder for this preprint (which was not certified by peer review) is the author/funder, who has granted medRxiv a license to display the preprint in perpetuity.

It is made available under a CC-BY 4.0 International license .

\section{Relevant conflicts of interest/financial disclosures:}

SF received consulting fees/honoraria for board membership from Retrophin Inc., Sun Pharma Advanced Research Co., LTD and Kashiv Pharma. CHW received research support from Sanofi, Biogen, Roche, consulting fees/honoraria from Amneal, Adamas, Impel, Kyowa, Mitsubishi, Neurocrine, US World Meds, Acadia, Acorda. RNA received consultation fees from Biogen, Denali, Genzyme/Sanofi and Roche. ZGO received consultancy fees from Lysosomal Therapeutics Inc. (LTI), Idorsia, Prevail Therapeutics, Inceptions Sciences (now Ventus), Ono Therapeutics, Denali and Deerfield. Rest of the authors have nothing to report.

\section{Funding}

This study was financially supported by grants from the Michael J. Fox Foundation, the Canadian Consortium on Neurodegeneration in Aging (CCNA), the Canada First Research Excellence Fund (CFREF), awarded to McGill University for the Healthy Brains for Healthy Lives initiative (HBHL), and Parkinson Canada. The Columbia University cohort is supported by the Parkinson's Foundation, the National Institutes of Health (K02NS080915, and UL1 TR000040) and the Brookdale Foundation. This research was supported in part by the Intramural Research Program of the NIH, National institute on Aging. 


\begin{abstract}
Background and objectives: The LRRK2 p.G2019S Parkinson's disease (PD) variant is associated with elevated glucocerebrosidase (GCase) activity in peripheral blood. We aimed to evaluate the association of other LRRK2 variants with PD and its association with GCase activity.
\end{abstract}

Methods: $L R R K 2$ and $G B A$ were fully sequenced in 1,123 PD patients and 576 controls from the Columbia and PPMI cohorts, in which GCase activity was measured in dried blood spots by liquid chromatography-tandem mass spectrometry.

Results: LRRK2 p.M1646T was associated with increased GCase activity in the Columbia University cohort $(\beta=1.58, p=0.0003)$, and increased but not significantly in the PPMI cohort $(\beta=0.29, p=0.58)$. p.M1646T was associated with $\mathrm{PD}(\mathrm{OR}=1.18,95 \% \mathrm{CI}=1.09-1.28, p=7.33 \mathrm{E}-05)$ in 56,306 PD patients and proxy-cases, and 1.4 million controls.

Conclusions: Our results suggest that the p.M1646T variant is associated with risk of PD with a small effect and with increased GCase activity in peripheral blood. 


\section{Introduction}

Parkinson's disease (PD) is mostly caused by an interaction between genetic and environmental factors. ${ }^{1}$ Variants in GBA and LRRK2 are among the most common genetic risk factors of PD. ${ }^{2,3}$ The frequency of these variants varies in different populations, with $G B A$ variants found in $5-20 \%{ }^{2}$ and LRRK2 variants reported in $1-40 \%$ of PD patients. ${ }^{4}$

The activity of the enzyme encoded by $G B A, \beta$-glucocerebrosidase (GCase), is reduced in carriers of $G B A$ variants, but also in a subset of PD patients without $G B A$ variants. ${ }^{5,6}$ There are contradicting results regarding the effect of the LRRK2 p.G2019S variant on GCase activity. In peripheral blood, this variant was associated with an increased activity, ${ }^{6}$ whereas in patient-derived dopaminergic neurons with $L R R K 2$ variants, GCase activity was reduced. ${ }^{7}$ A variant in TMEM175, p.T393M, has been associated with reduced GCase activity and, together with $G B A$ variants and the LRRK2 p.G2019S variant, explain only $23 \%$ of the variance in GCase activity in peripheral blood. ${ }^{8}$ These observations suggest that other genetic or environmental factors affect GCase activity.

In the current study, we performed full sequencing of $L R R K 2$ and $G B A$ and examined the effect of common LRRK2 variants on GCase activity in peripheral blood in two cohorts: from Columbia University and from the Parkinson's Progression Markers Initiative (PPMI). We further examined the association of $L R R K 2$ variants identified through this analysis with risk of PD using data from the International Parkinson's Disease Genomics Consortium (IPDGC), UK biobank and 23andMe, Inc. genome-wide association study (GWAS) meta-analysis. ${ }^{9}$ 


\section{Materials and methods}

\section{Study population}

To analyze the effects of LRRK2 variants on GCase activity, two cohorts were included: 1) The Columbia University cohort $(\mathrm{n}=1,229, \mathrm{PD}=797$, Controls=432) and 2) The PPMI cohort $(\mathrm{n}=470$, $\mathrm{PD}=326$, Controls=144). Both cohorts have been previously described, ${ }^{10,11}$ and their demographic data is detailed in Table 1. The Columbia cohort consisted of patients and controls of mixed ethnicity (mainly of European origin, including 308 individuals of Ashkenazi Jewish descent). Data on the effect of the LRRK2 p.M1646T variant on risk of PD was extracted from the recent PD GWAS, including 37,688 PD patients, 18,618 UK Biobank proxy-cases and 1.4 million controls. ${ }^{9}$ All PD patients were diagnosed by movement disorder specialists according to the UK brain bank criteria ${ }^{12}$ or the MDS clinical diagnostic criteria. ${ }^{13}$

\section{Standard Protocol Approvals, Registrations, and Patient Consents}

The institutional review boards approved the study protocols, and informed consent was obtained from all participants before entering the study. 23andMe participants provided informed consent and participated in the research online, under a protocol approved by the external AAHRPP-accredited IRB, Ethical \& Independent Review Services (E\&I Review).

\section{Genetic analysis}

LRRK2 and GBA Sequencing in the Columbia University cohort

We performed full sequencing of $L R R K 2$ and $G B A$ in the Columbia University cohort using targeted sequencing with Molecular Inversion Probes (MIPs) and Sanger sequencing as previously described. ${ }^{14-16}$ The full protocol and the library of MIPs used for sequencing LRRK2 and GBA are available online (https://github.com/gan-orlab/MIP_protocol). A standard quality control protocol 
was performed as previously described, ${ }^{17}$ and the code is available at https://github.com/ganorlab/MIPVar/.

Genetic data from PPMI and IPDGC

Due to the alignment difficulties with $G B A$, data on $G B A$ variants in the PPMI cohort were extracted from combined data including whole genome sequencing data, whole exome sequencing data and RNA-seq as previously reported. ${ }^{11}$ Data on LRRK2 p.G2019S, p.M1646T, p.N551K-p.R1398H and p.N2081D were extracted from imputed GWAS data (Illumina Immunochip and NeuroX arrays) downloaded from the PPMI project website (https://ida.loni.usc.edu/). To examine the association of LRRK2 variants with PD, we extracted data from the recent PD GWAS meta-analysis. ${ }^{9}$

\section{GCase activity}

Dried blood spots (DBS) were obtained as previously described. ${ }^{18,19}$ GCase activity was measured in participants from Columbia University at Sanofi laboratories by liquid chromatography-tandem mass spectrometry (LC-MS/MS) from dried blood spots, as a part of multiplex assay with four additional lysosomal enzymes as previously described. ${ }^{6,20}$ PPMI study participants donated blood on the first visit (baseline) and every year, which was frozen and stored in -80C freezer. Samples from the first three years of the cohort were thawed, and DBS were obtained. Activity was measured as previously described, using the mean GCase activity for each participant across all visits. ${ }^{6,11}$

\section{Statistical Analysis}

Linear regression models were used to test for association between LRRK2 variants and GCase activity in the Columbia and PPMI cohorts, adjusting for age, sex, PD status, GBA status and ethnicity. In the PPMI cohort additional adjustment for white blood cells count was performed as suggested previously. ${ }^{11}$ We then repeated the analysis after excluding LRRK2 p.G2019S and GBA variants carriers in both Columbia and PPMI cohorts. In addition, to examine whether there are sex- 
specific effects, we performed additional analyses stratifying the cohorts by sex (code available at https://github.com/gan-orlab/LRRK2_GCase). Bonferroni correction for multiple comparisons was applied as needed. All statistical analyses were performed using R or PLINK version 1.9. ${ }^{21,22}$

\section{Results}

In the Columbia University cohort, we identified 26 LRRK2 common variants with MAF >1\% (Supplementary Table 1), including 9 nonsynonymous variants, 12 intronic variants and 5 synonymous variants. We did not analyze rare variants, as the small number of carriers of these variants would not allow estimating their impact on GCase activity.

The LRRK2 p.M1646T variant was associated with increased GCase activity compared to non-carriers (12.65 mmol/l/h vs. $11.38 \mathrm{mmol} / \mathrm{l} / \mathrm{h}$, respectively, $\beta=1.58, p=0.0003$, Table 2, Supplementary Table 1) in the Columbia University cohort. The effect of p.M1646T on GCase activity was stronger in $\mathrm{PD}(\mathrm{GCase}=13.08 \mathrm{mmol} / \mathrm{l} / \mathrm{h}, \beta=1.74, p=0.0011)$ and did not reach statistical significance in controls $(\mathrm{GCase}=11.96 \mathrm{mmol} / \mathrm{l} / \mathrm{h}, \beta=1.37, p=0.068$, Table 2, Supplementary Table 23). After exclusion of p.G2019S carriers, the association of p.M1646T with increased activity remained strong (GCase $=12.64 \mathrm{mmol} / \mathrm{l} / \mathrm{h}, \beta=1.73, p=6.24 \mathrm{E}-05$, Supplementary Table 4-5). The LRRK2 p.G2019S variant was associated with increased GCase activity as previously described. ${ }^{6}$ Two variants from the protective haplotype p.N551K-p.R1398H-p.K1423K were nominally associated with GCase activity, but this association was not statistically significant after Bonferroni correction (Table 2). We then demonstrated that the LRRK2 p.M1646T variant was strongly associated with PD, using data from the recent PD GWAS meta-analysis, ${ }^{9}$ including 37,688 PD patients, 18,618 UK Biobank proxy-cases and 1.4 million controls $\quad(\mathrm{OR}=1.1895 \% \mathrm{CI}=1.09-1.28$, $p=7.33 \mathrm{E}-05)$.

As a replication for GCase activity, we used data from the PPMI cohort, and analyzed the association of p.R1398H (representing the protective haplotype), p.M1646T, p.G2019S and 
p.N2081D with GCase activity (Table 2, Supplementary Table 8). The p.M1646T variant showed the same direction of effect and similar average GCase activity value as observed in the Columbia cohort, but did not reach statistical significance, possibly due to the small number of carriers $(\mathrm{n}=23)$, compared to non-carriers $(12.72 \mathrm{mmol} / \mathrm{l} / \mathrm{h}$ vs. $11.84 \mathrm{mmol} / \mathrm{l} / \mathrm{h}$, respectively, $\beta=0.29, p=0.59$; Table 2). Only six carriers of the LRRK2 p.G2019S variant were included in the PPMI cohort, and the association of this variant with GCase activity, as well as of the protective haplotype, were not statistically significant after Bonferroni correction (Table 2, Supplementary Table 8). Stratified analysis by sex did not identify sex differences in GCase activity in both cohorts (Supplementary Table 6-8). 


\section{Discussion}

In the current study, we show that the LRRK2 p.M1646T variant is associated with PD and with increased GCase activity in peripheral blood. The association of this variant with PD has been previously demonstrated, ${ }^{3,23}$ and we now confirmed it in a larger European cohort. Despite its smaller effect on PD risk compared to the LRRK2 p.G2019S variant, the effect of p.M1646T on GCase activity was larger than the effect of p.G2019S. However, since the results on GCase activity did not fully replicate in the PPMI cohort, additional studies are required to understand the associations between $L R R K 2$ variants, GCase activity and PD risk.

In a recent study, the LRRK2 pathogenic variants p.G2019S, p.R1441G, and p.R1441C were associated with reduced GCase activity in patient-derived dopaminergic neurons, and correction of these variants resulted in normalization of GCase activity. ${ }^{24}$ Conversely, in the current study, deleterious LRRK2 variants (p.G2019S and p.M1646T) were associated with increased GCase activity in peripheral blood. There are several potential explanations for these differences in the direction of effects on GCase activity, including: a) different effects of $L R R K 2$ variants in the central nervous system vs. peripheral blood, b) the possibility that iPSC-derived dopaminergic neurons, which are young cells, are different than patient tissues, due to the natural aging process, and c) the different methods used to measure GCase activity.

Considering the study suggesting that $L R R K 2$ variants are associated with reduced GCase activity, ${ }^{24}$ drugs targeting $L R R K 2$ activity could be repurposed for GBA-PD, and drugs that target GCase activity could be used for LRRK2-PD. However, this potential association between $L R R K 2$, GBA and GCase activity should be carefully studied further, since other data suggests that LRRK2 variants are not associated with reduced GCase activity. Patients with GBA-PD (and thus, reduced GCase activity) have a more severe phenotype with faster disease progression and cognitive decline, depression and anxiety, compared to sporadic PD. ${ }^{25-27}$ In contrast, LRRK2 variants carriers have a 
milder phenotype with slower disease progression and lower frequency of cognitive symptoms compared to sporadic PD. ${ }^{28,} 29$ Moreover, two independent studies demonstrated that carriers of both LRRK2 and GBA variants seem to have a benign phenotype, similar to those who carry LRRK2 variants only. ${ }^{30,31}$ If indeed LRRK2 variants lead to reduced GCase activity as suggested, ${ }^{24}$ we would expect that patients with both $L R R K 2$ and $G B A$ variants would have a severe phenotype. Instead,

their phenotype is milder, ${ }^{30,31}$ which may raise the hypothesis that the increased GCase activity we observed in peripheral blood may have some protective effect on PD phenotype. This hypothesis requires additional studies in human cohorts and disease models.

Our study has several limitations. In our cohorts, difference in sex between PD patients and controls was significant. To address this limitation, we adjusted the regression model with sex as covariate, as well as other covariates. The Columbia cohort included individuals of mixed ethnicity, mainly of European and Ashkenazi Jewish ancestry, and we adjusted the regression models for ethnicity. Another limitation is that GCase activity was measured in blood, which does not necessarily reflect GCase activity in the brain. More specifically, in the PPMI cohort it was measured from frozen blood, and the total number of carriers of $L R R K 2$ variants in this cohort was relatively low.

To conclude, we demonstrated that the LRRK2 p.M1646T variant is associated with increased GCase activity in peripheral blood and with increased risk of PD. The interplay between $L R R K 2$, $G B A$ and GCase activity should be studied in additional cohorts and relevant disease models.

\section{Acknowledgements}

Data used in the preparation of this article were obtained from the Parkinson's Progression Markers Initiative (PPMI) database (www.ppmiinfo.org/data). For up-to-date information on the study, visit www.ppmiinfo.org." "PPMI - a public-private partnership - is funded by the Michael J. Fox 
Foundation for Parkinson's Research and funding partners, including (list the full names of all of the PPMI funding partners found at www.ppmiinfo.org/fundingpartners). We would also like to thank the research participants and employees of 23andMe for making this work possible. The full GWAS summary statistics for the 23 andMe discovery data set will be made available through 23 andMe to qualified researchers under an agreement with 23andMe that protects the privacy of the 23andMe participants. Please visit research.23andme.com/collaborate/ for more information and to apply to access the data. We would like to also thank all members of the International Parkinson Disease Genomics Consortium (IPDGC). For a complete overview of members, acknowledgements and funding, please see http://pdgenetics.org/partners. ZGO is supported by the Fonds de recherche du Québec - Santé (FRQS) Chercheurs-boursiers award, in collaboration with Parkinson Quebec, and by the Young Investigator Award by Parkinson Canada. The access to part of the participants for this research has been made possible thanks to the Quebec Parkinson's Network (http://rpq-qpn.ca/en/). $\mathrm{KS}$ is supported by a post-doctoral fellowship from the Canada First Research Excellence Fund (CFREF), awarded to McGill University for the Healthy Brains for Healthy Lives initiative (HBHL). We thank Daniel Rochefort, Hélène Catoire, Clotilde Degroot and Vessela Zaharieva for their assistance.

\section{Authors' Roles}

1) Research project: A. Conception (YLS, ZGO, KS), B. Organization (YLS, EY, LK, UR, JAR, FA, KM, SBL, DS, SF, CW, SBC, RNA, ZGO, KS), C. Execution (YLS, EY, UR, LK, KM, JAR, FA, DS, KS).

2) Statistical Analysis: A. Design (YLS, ZGO, KS), B. Execution (YLS, KS).

3) Manuscript Preparation: A. Writing of the first draft (YLS, ZGO, KS), B. Review and Critique (YLS, EY, LK, UR, JAR, FA, KM, SBL, DS, SF, CW, SBC, PS, RNA, ZGO, KS). 
medRxiv preprint doi: https://doi.org/10.1101/2020.09.23.20197558; this version posted September 24, 2020. The copyright holder for this preprint (which was not certified by peer review) is the author/funder, who has granted medRxiv a license to display the preprint in perpetuity. It is made available under a CC-BY 4.0 International license .

\section{Financial Disclosures of all authors (for the preceding 12 months):}

SF received consulting fees/honoraria for board membership from Retrophin Inc., Sun Pharma Advanced Research Co., LTD and Kashiv Pharma. CHW received research support from Sanofi, Biogen, Roche, consulting fees/honoraria from Amneal, Adamas, Impel, Kyowa, Mitsubishi, Neurocrine, US World Meds, Acadia, Acorda. RNA received consultation fees from Biogen, Denali, Genzyme/Sanofi and Roche. ZGO received consultancy fees from Lysosomal Therapeutics Inc. (LTI), Idorsia, Prevail Therapeutics, Inceptions Sciences (now Ventus), Ono Therapeutics, Denali and Deerfield. Rest of the authors have nothing to report. 


\section{References}

1. Ascherio A, Schwarzschild MA. The epidemiology of Parkinson's disease: risk factors and prevention. The Lancet Neurology 2016;15(12):1257-1272.

2. Gan-Or Z, Amshalom I, Kilarski LL, et al. Differential effects of severe vs mild GBA mutations on Parkinson disease. Neurology 2015;84(9):880-887.

3. Ross OA, Soto-Ortolaza Al, Heckman MG, et al. Association of LRRK2 exonic variants with susceptibility to Parkinson's disease: a case-control study. Lancet Neurol 2011;10(10):898-908.

4. Healy DG, Falchi M, O'Sullivan SS, et al. Phenotype, genotype, and worldwide genetic penetrance of LRRK2-associated Parkinson's disease: a case-control study. Lancet Neurol 2008;7(7):583-590.

5. Gegg ME, Burke D, Heales SJ, et al. Glucocerebrosidase deficiency in substantia nigra of parkinson disease brains. Annals of neurology 2012;72(3):455-463.

6. Alcalay RN, Levy OA, Waters CC, et al. Glucocerebrosidase activity in Parkinson's disease with and without GBA mutations. Brain 2015;138(Pt 9):2648-2658.

7. Ysselstein D, Nguyen M, Young TJ, et al. LRRK2 kinase activity regulates lysosomal glucocerebrosidase in neurons derived from Parkinson's disease patients. Nature communications 2019;10(1):1-9.

8. Krohn L, Öztürk TN, Vanderperre B, et al. Genetic, structural, and functional evidence link TMEM175 to synucleinopathies. Annals of Neurology 2020;87(1):139-153.

9. Nalls $\mathrm{MA}$, Blauwendraat $\mathrm{C}$, Vallerga $\mathrm{CL}$, et al. Identification of novel risk loci, causal insights, and heritable risk for Parkinson's disease: a meta-analysis of genome-wide association studies. Lancet Neurol 2019;18(12):1091-1102.

10. Alcalay RN, Levy OA, Wolf $P$, et al. SCARB2 variants and glucocerebrosidase activity in Parkinson's disease. npj Parkinson's Disease 2016;2(1):1-4.

11. Alcalay RN, Wolf $P$, Chiang MSR, et al. Longitudinal Measurements of Glucocerebrosidase activity in Parkinson's patients. Annals of Clinical and Translational Neurology 2020;.

12. Hughes AJ, Daniel SE, Kilford L, Lees AJ. Accuracy of clinical diagnosis of idiopathic Parkinson's disease: a clinico-pathological study of 100 cases. J Neurol Neurosurg Psychiatry 1992;55(3):181-184.

13. Postuma RB, Berg D, Stern M, et al. MDS clinical diagnostic criteria for Parkinson's disease. Movement disorders 2015;30(12):1591-1601.

14. Ruskey JA, Greenbaum L, Roncière L, et al. Increased yield of full GBA sequencing in Ashkenazi Jews with Parkinson's disease. European journal of medical genetics 2019;62(1):65-69.

15. Ross JP, Dupre N, Dauvilliers Y, et al. Analysis of DNAJC13 mutations in French-Canadian/French cohort of Parkinson's disease. Neurobiol Aging 2016;45:212.e213-212.e217.

16. Ouled Amar Bencheikh B, Leveille E, Ruskey JA, et al. Sequencing of the GBA coactivator, Saposin C, in Parkinson disease. Neurobiol Aging 2018;72:187 e181-187 e183.

17. Rudakou U, Ruskey JA, Krohn L, et al. Analysis of common and rare VPS13C variants in late-onset Parkinson disease. Neurology Genetics 2020;6(1).

18. Olivova $\mathrm{P}$, Cullen $\mathrm{E}$, Titlow $\mathrm{M}$, et al. An improved high-throughput dried blood spot screening method for Gaucher disease. Clin Chim Acta 2008;398(1-2):163-164.

19. Reuser AJ, Verheijen FW, Bali D, et al. The use of dried blood spot samples in the diagnosis of lysosomal storage disorders--current status and perspectives. Mol Genet Metab 2011;104(1-2):144-148.

20. Zhang XK, Elbin CS, Chuang W-L, et al. Multiplex enzyme assay screening of dried blood spots for lysosomal storage disorders by using tandem mass spectrometry. Clinical chemistry 2008;54(10):1725-1728. 21. Purcell S, Neale B, Todd-Brown K, et al. PLINK: a tool set for whole-genome association and population-based linkage analyses. Am J Hum Genet 2007;81(3):559-575.

22. Chang CC, Chow CC, Tellier LC, Vattikuti S, Purcell SM, Lee JJ. Second-generation PLINK: rising to the challenge of larger and richer datasets. Gigascience 2015;4:7.

23. Heckman MG, Soto-Ortolaza Al, Aasly JO, et al. Population-specific frequencies for LRRK2 susceptibility variants in the Genetic Epidemiology of Parkinson's Disease (GEO-PD) Consortium. Mov Disord 2013;28(12):1740-1744. 
medRxiv preprint doi: https://doi.org/10.1101/2020.09.23.20197558; this version posted September 24, 2020. The copyright holder for this preprint (which was not certified by peer review) is the author/funder, who has granted medRxiv a license to display the preprint in perpetuity.

It is made available under a CC-BY 4.0 International license .

24. Ysselstein D, Nguyen M, Young TJ, et al. LRRK2 kinase activity regulates lysosomal glucocerebrosidase in neurons derived from Parkinson's disease patients. Nat Commun 2019;10(1):5570. 25. Liu G, Boot B, Locascio JJ, et al. Specifically neuropathic Gaucher's mutations accelerate cognitive decline in Parkinson's. Ann Neurol 2016;80(5):674-685.

26. Swan M, Doan N, Ortega RA, et al. Neuropsychiatric characteristics of GBA-associated Parkinson disease. Journal of the neurological sciences 2016;370:63-69.

27. Cilia R, Tunesi S, Marotta G, et al. Survival and dementia in GBA-associated Parkinson's disease: The mutation matters. Ann Neurol 2016;80(5):662-673.

28. Piredda R, Desmarais $P$, Masellis M, Gasca-Salas C. Cognitive and psychiatric symptoms in genetically determined Parkinson's disease: a systematic review. European journal of neurology 2020;27(2):229-234.

29. Alcalay RN, Mejia-Santana H, Mirelman A, et al. Neuropsychological performance in LRRK2 G2019S carriers with Parkinson's disease. Parkinsonism \& related disorders 2015;21(2):106-110.

30. Yahalom G, Greenbaum L, Israeli-Korn S, et al. Carriers of both GBA and LRRK2 mutations, compared to carriers of either, in Parkinson's disease: Risk estimates and genotype-phenotype correlations.

Parkinsonism Relat Disord 2019;62:179-184.

31. Omer N, Giladi N, Gurevich T, et al. A Possible Modifying Effect of the G2019S Mutation in the LRRK2 Gene on GBA Parkinson's Disease. Mov Disord 2020. 
medRxiv preprint doi: https://doi.org/10.1101/2020.09.23.20197558; this version posted September 24, 2020. The copyright holder for this preprint (which was not certified by peer review) is the author/funder, who has granted medRxiv a license to display the preprint in perpetuity.

It is made available under a CC-BY 4.0 International license .

Table 1. Demographic data of the cohorts to study LRRK2 effect on GCase activity.

\begin{tabular}{|c|c|c|c|c|c|c|}
\hline Cohort & PD & Controls & $\begin{array}{c}\text { PD age } \\
\text { (mean, SD } \\
\text { in years) }\end{array}$ & $\begin{array}{c}\text { Controls } \\
\text { age (mean, } \\
\text { SD in years) }\end{array}$ & $\begin{array}{c}\text { PD males } \\
\text { (N, } \\
\text { percentage) }\end{array}$ & $\begin{array}{c}\text { Controls } \\
\text { males (N, } \\
\text { percentage) }\end{array}$ \\
\hline Columbia & 797 & 432 & $\begin{array}{c}65.80 \\
(11.04)\end{array}$ & $64.75(9.94)$ & $512(64 \%)$ & $116(27 \%)$ \\
\hline & & & & 61.37 & & \\
PPMI & 326 & 144 & $60.12(9.67)$ & $(10.91)$ & $216(66 \%)$ & $100(69 \%)$ \\
\hline
\end{tabular}

PD, Parkinson's disease; SD, standard deviation; Columbia, cohort from Columbia University, NY; PPMI,

Parkinson's Progression Markers Initiative cohort. 
Table 2. Impact of LRRK2 variants on GCase activity.

\begin{tabular}{|c|c|c|c|c|c|c|}
\hline LRRK2 variants & $\mathbf{N}$ of carriers & Estimate & SE & p-value* & GCase_mean & Gcase_SD \\
\hline \multicolumn{7}{|c|}{ Columbia cohort } \\
\hline \multicolumn{7}{|c|}{ PD + controls $(\mathrm{N}=1229)$} \\
\hline p.R1398H & 204 & 0.521 & 0.233 & 0.026 & 11.960 & 3.804 \\
\hline p.M1646T & 58 & 1.578 & 0.431 & 0.0003 & 12.652 & 4.529 \\
\hline p.G2019S & 61 & 1.370 & 0.438 & 0.0018 & 12.798 & 4.340 \\
\hline \multicolumn{7}{|c|}{ PD $(\mathrm{N}=797)$} \\
\hline p.R1398H & 123 & 0.495 & 0.298 & 0.097 & 11.776 & 3.863 \\
\hline p.M1646T & 36 & 1.736 & 0.528 & 0.0011 & 13.078 & 4.600 \\
\hline p.G2019S & 57 & 1.440 & 0.450 & 0.0014 & 12.877 & 4.471 \\
\hline \multicolumn{7}{|c|}{ Controls $(\mathrm{N}=432)$} \\
\hline p.R1398H & 81 & 0.755 & 0.383 & 0.050 & 12.240 & 3.720 \\
\hline p.M1646T & 22 & 1.367 & 0.746 & 0.068 & 11.956 & 4.425 \\
\hline p.G2019S & 4 & -0.030 & 1.719 & 0.986 & 11.658 & 1.286 \\
\hline \multicolumn{7}{|c|}{ PPMI cohort } \\
\hline \multicolumn{7}{|c|}{$P D+$ controls $(N=470)$} \\
\hline p.R1398H & 61 & -0.724 & 0.340 & 0.034 & 10.936 & 2.961 \\
\hline p.M1646T & 23 & 0.295 & 0.543 & 0.587 & 12.717 & 3.510 \\
\hline p.G2019S & 6 & 0.004 & 1.050 & 0.997 & 11.453 & 2.648 \\
\hline \multicolumn{7}{|c|}{ PD $(\mathrm{N}=326)$} \\
\hline p.R1398H & 41 & -0.893 & 0.406 & 0.028 & 10.445 & 3.045 \\
\hline p.M1646T & 17 & 0.073 & 0.623 & 0.907 & 12.385 & 3.259 \\
\hline p.G2019S & 6 & -0.078 & 1.037 & 0.940 & 11.453 & 2.648 \\
\hline \multicolumn{7}{|c|}{ Controls $(\mathrm{N}=144)$} \\
\hline p.R1398H & 20 & -0.240 & 0.622 & 0.701 & 11.941 & 2.564 \\
\hline p.M1646T & 6 & 1.169 & 1.082 & 0.282 & 13.657 & 4.333 \\
\hline
\end{tabular}

SE, standard error; N, number; GCase_mean, mean glucocerebrosidase activity, $\mu$ mol/1/h; SD, standard deviation; PD, Parkinson's disease; Columbia, cohort from Columbia University, NY; PPMI, Parkinson's

Progression Markers Initiative cohort; *, Bonferroni correction significance threshold for Columbia cohort $(\alpha=0.05 / 26=0.0019)$ and $(\alpha=0.05 / 5=0.01)$ for PPMI cohort. 